\title{
Editorial
}

\section{Opportunities and challenges in targeting genes for therapy}

Recent progress in mammalian gene targeting has now advanced the scope of site-specific alteration of genomic DNA from the realm of mouse embryonic stem (ES) cells to that of human somatic cells. ${ }^{1-3}$ The implications of these advances for development of novel gene therapy strategies were discussed in Paris, in October 1997 at the 'Gene Therapy: The Next Generation' meeting. Of the potential strategies discussed at the meeting, modification of genomic loci by gene targeting appears to have the most immediate therapeutic application. In particular, the targeting of genomic DNA with RNA/DNA chimeric oligonucleotides or with small DNA fragments has made important strides. ${ }^{1,3}$

The use of gene targeting approaches over the cDNAbased gene therapy strategies has several advantages. The most relevant is that the relationship between the coding and regulatory elements of the targeted gene is maintained. Only by maintaining this level of genomic integrity can one be assured that the targeted gene will be appropriately expressed in an individual cell. Such cellappropriate expression will be critical both for long-term maintenance and for ensuring that there is no additional pathology that may be the result of long-term cell-inappropriate expression. Another major advantage of recently developed gene targeting strategies has been the relatively high efficiency of targeted replacement. ${ }^{1-3}$ Generally, efficiencies between 1 and $10 \%$ have been observed. When considering the treatment of diseases where $100 \%$ corrected function is not necessary to restore normal phenotype, these gene targeting strategies have a distinct appeal.

Site-specific alteration of genomic sequences by small fragments of DNA, ie small fragment homologous replacement (SFHR), has shown promise for treatment of cystic fibrosis $(\mathrm{CF})^{1,4-6}$ and may have direct applications to gene therapy of other inherited disorders. ${ }^{1}$ The target locus for SFHR of CF was a 3-bp deletion in exon 10 of the CF transmembrane conductance regulator (CFTR) gene that results in deletion of phenylalanine at codon $508(\Delta$ F508). SFHR-mediated replacement has been used to correct transformed ${ }^{4-6} \mathrm{CF}$ airway epithelial cells. In addition to the insertion of 3-bp, SFHR has proven effective for site-specific deletion. The $\Delta$ F508 sequence has been introduced into primary normal human airway epithelial cells. ${ }^{4}$ Moreover, studies in mouse ES cells and mouse airway epithelial cells $s^{4,7}$ have given rise to isogenic cell clones carrying $\Delta$ F508 CFTR. These studies indicate that at least 3-bp can be deleted or inserted in a site-specific fashion, and that this deletion or insertion can occur concomitant with a single conversion within 100-bp of the insertion/deletion site.

The mechanisms that underlie SFHR and other gene targeting strategies are not clearly defined or understood. There are several enzymatic pathways that might potentially play a role in site-specific targeted replacement. These include, but are not limited to, mismatch repair, excision repair, homologous recombination, gene conversion, replication-dependent strand displacement, and transcription-dependent nucleotide exchange. 1,3,9 Further elucidation of these underlying mechanisms is paramount for optimization of therapy and defining the best therapeutic route.

Gene targeting strategies rely either on artificial delivery systems or on the adeno-associated virus (AAV). ${ }^{2}$ The host immune response normally associated with the viral vector systems now employed for gene therapy, does not appear to be a factor that will undermine gene targeting. However, further studies will be necessary to address this issue. What also needs to be evaluated is the longterm pharmacological impact of the artificial delivery system on the cellular metabolism. If the delivery systemnucleic acid complex activates an apoptotic cascade ${ }^{10}$ leading to in vivo cell death, the therapeutic efficacy of the gene targeting protocol will be compromised. It will therefore be imperative that the delivery system not only be studied in terms of its ability to deliver the nucleic acid to the cells, but also in terms of how it will impact the long-term cell viability.

While these techniques show clinical promise, the cells expressing the targeted gene might not readily lend themselves to analysis. Another aspect of gene therapy strategies that involves gene targeting will be assessment of functional efficacy. The observed frequency of gene targeting in cultured cells is orders of magnitude higher than that observed for classical homologous recombination. However, it has been difficult to extrapolate accurately the degree to which this approach will be effective for targeting in somatic cells in vivo. It is therefore necessary to develop both model systems and/or assay techniques for in vitro and in vivo assessment of gene targeting efficacy. Selectable marker and reporter genes with inactivating mutations have already been used to gain some insight into gene targeting efficacy. One recent study measuring the gene targeting efficiency of AAV used the aminoglycoside phosphotransferase $\left(\right.$ neo $\left.^{\mathrm{r}}\right)$ and hypoxanthine phosphoribosyl transferase (HPRT) genes. ${ }^{2}$ Studies investigating the efficiency of targeted correction with RNA/DNA chimeric oligonucleotides have used the tetracycline resistance gene ${ }^{11}$ and alkaline phosphatase 
gene. ${ }^{12}$ Studies investigating SFHR-mediated targeting have used the Zeocin resistance gene. ${ }^{13}$

Along with defining the appropriate model system for assessment of parameters that influence the efficiency of homologous replacement, it will be critical to assess whether, and to what extent, homologous replacement has occurred in a given tissue in vivo. Verification of homologous replacement in chromosomal DNA generally involves screening of DNA from a population of cells for targeted exchange by polymerase chain reaction (PCR) amplification. PCR has been a powerful tool for analysis of gene targeting that is highly dependent on primer design and in defining amplification conditions. ${ }^{1,5,6,14}$ Allele-specific primers have been developed that facilitate the process of differentiating between wild-type and mutant alleles of CFTR. ${ }^{1,5,14}$ An in situ allele-specific reverse-transcriptase PCR (RT-PCR) analysis of fixed tissue sections and cultured cells has been effective in identifying individual cells expressing specific CFTR alleles. $^{15}$

Determination of the gene targeting efficacy will ultimately require detection of a functional protein. If it is possible to differentiate between the presence of the mutant and the wild-type form of the proteins, antibodies can be employed. However, functional/phenotypic analysis will provide the definitive determination of the efficacy of homologous replacement.

The specific application of any one gene targeting strategy to the treatment of a given disease will depend on a variety of factors that include: the target organ/cell type, the physical barriers to cellular delivery, the efficiency necessary to achieve correction of the disease pathology, and the nature of the mutation. Of these considerations, the most likely to impact the choice of targeting strategy are the target organ/cell type and the nature of the mutation. The different gene targeting approaches may depend on specific enzymatic pathways that are independent of each other, both in their predominance in different cell types and in their ability to resolve specific genetic lesions. For a target cell to resolve a specific genetic lesion may require proliferative or transcriptional states depending on the gene targeting strategy employed.

Furthermore, some approaches will correct multiple bases (eg SFHR), while others appear to be limited to resolving single bases. Paramount to making an informed choice as to which targeting approach is optimal, is an evaluation of the limits of non-homology that the correcting DNA can accommodate before the efficiency of the technique is no longer therapeutically relevant.

While concern about the level of random integration associated with the various gene targeting strategies has been expressed, there is no indication, at present, that significant levels occur. Southern hybridization and sequence analysis of mouse ES cell clones and clones of human airway epithelial cells carrying a corrected selectable marker gene has not revealed any non-targeted integration following SFHR. ${ }^{16}$ It should also be noted that gene targeting with oligonucleotides does not involve the introduction of regulatory and/or polyadenylation signal sequences associated with expression vector-based systems. Such sequences have a greater potential for influencing endogenous, non-targeted genes within or near the integration site in terms of modifying their expression.
Development of effective gene targeting methodologies has been encouraging. ${ }^{1,3,9}$ However, before such approaches can be successfully and effectively implemented in the clinic, a clear understanding of the cellular and molecular mechanisms that underlie uptake of DNA fragments and the homologous exchange process is imperative. It is only through such insight that gene targeting strategies for therapy of inherited disorders can be optimized to be efficacious.

DC Gruenert
Gene Therapy Core Center
Cardiovascular Research Institute
Departments of Laboratory Medicine and Stomatology
University of California San Francisco
San Francisco, CA 94143, USA

\section{References}

1 Gruenert DC. Gene correction with small DNA fragments. Curr Res Molec Therapeut: Gene Ther Oligonucl 1998; 1: 607-613.

2 Russell DW, Hirata RK. Human gene targeting by viral vectors. Nat Genet 1998; 18: 325-330.

3 Yanez RJ, Porter ACG. Therapeutic gene targeting. Gene Therapy 1998; 5: 149-159.

4 Goncz KK et al. Prospects for gene therapy by gene targeting. Am Soc Gene Ther Mtg 1998 (Abstr.).

5 Goncz KK, Gruenert DC. Site-directed alteration of DNA by small fragment homologous replacement (SFHR). In: Kmiec EB (ed). Gene Targeting Vector Protocols. Humana Press: Totowa, NJ 1999, pp 85-99.

6 Kunzelmann $\mathrm{K}$ et al. Gene targeting of CFTR DNA in CF epithelial cells. Gene Therapy 1996; 3: 859-867.

$7 \mathrm{Xu}$ ZD et al. Generation of mouse embryonic stem cells carrying the CFTR $\triangle$ F508 mutation by small fragment targeted gene replacement. Pediat Pulmonol 1993; (Suppl. 9): 14A (Abstr.).

$8 \mathrm{Xu} \mathrm{Z}$ et al. Transgenic CF animal development by small fragment homologous replacement. Am J Hum Genet 1996; 59: A199 (Abstr. 1135).

9 Woolf TM. Therapeutic repair of mutated nucleic acid sequences. Nat Biotechnol 1998; 16: 341-344.

10 Ebert $\mathrm{O}$ et al. Lymphocyte apoptosis: induction by gene transfer techniques. Gene Therapy 1997; 4: 296-302.

11 Cole-Strauss A et al. Targeted gene repair directed by the chimeric RNA/DNA oligonucleotide in a mammalian cell-free extract. Nucleic Acids Res 1999; 27: 1323-1330.

12 Yoon K, Cole-Strauss A, Kmiec EB. Targeted gene correction of episomal DNA in mammalian cells mediated by a chimeric RNA-DNA oligonucleotide. Proc Natl Acad Sci USA 1996; 93: 2071-2076.

13 Colosimo A et al. Gene targeting of episomal DNA in CF and non-CF epithelial cells. Pediat Pulmonol 1998; (Suppl. 17): 234 (Abstr.).

14 Goncz KK, Kunzelmann K, Xu Z, Gruenert DC. Targeted replacement of normal and mutant CFTR sequences in human airway epithelial cells using DNA fragments. Hum Mol Genet 1998; 7: 1913-1919.

15 Dohrman A, Goncz KK, Gruenert DC. Differential detection of wild-type and $\triangle$ F508 CFTR by allele-specific in situ RT-PCR. Pediat Pulmonol 1995; (Suppl. 12): LB22 (Abstr.).

\section{Reference added in proof}

16 Colosimo A et al. Gene targeting on expression vector systems mediated by SFHR technique. American Society of Gene Therapy, 2nd Annual Meeting, 9-13 June 1999, Washington DC, p 119a. 